REPORTE DE CASO

\title{
Primer reporte de un caso de ehrlichiosis monocítica canina en la provincia de Santa Fe, Argentina
}

\author{
Tarragona $\mathrm{EL}^{1,2^{*}}$, Flores $\mathrm{FS}^{3}$, Herrera $\mathrm{CL}^{4}$, Dalinger $\mathrm{M}^{4}$, Aguirre $\mathrm{N}^{1}$, Monje $\mathrm{LD}^{3}$, Nava $\mathrm{S}^{1,2}$ \\ ${ }^{1}$ Laboratorio de Parasitología e Inmunología, Estación Experimental Agropecuaria Rafaela, Instituto \\ Nacional de Tecnología Agropecuaria (INTA), Argentina. \\ 2 Consejo Nacional de Investigaciones Científicas y Técnicas (CONICET), Argentina. \\ ${ }^{3}$ Laboratorio de Ecología de Enfermedades, Instituto de Ciencias Veterinarias del Litoral \\ (Universidad Nacional del Litoral - CONICET), Esperanza, Santa Fe, Argentina. \\ ${ }^{4}$ Actividad privada.
}

* Correspondencia: Evelina L. Tarragona, INTA EEA Rafaela, ruta 34 km 227, CP 2300, Rafaela, Santa Fe, Argentina.

E-mail: tarragona.evelina@inta.gob.ar

RESUMEN. La ehrlichiosis monocitica canina (EMC) es una enfermedad causada por la bacteria Ehrlichia canis, de distribución mundial, alta mortalidad en caninos doméstico y síntomas inespecíficos, lo que dificulta su diagnóstico clínico. Ehrlichia canis es transmitida por la garrapata Rhipicephalus sanguineus sensu lato a un hospedador, en Argentina se reconocen dos linajes (tropical y sensu stricto) de dicha especie. El objetivo del presente trabajo es reportar el primer caso confirmado de EMC por $E$. canis en un canino de la ciudad de Rafaela, Santa Fe, área endémica de R. sanguineus s.s. El 18/02/2019 llegó a la consulta privada un canino con síntomas inespecíficos como hipertermia tarde/noche, depresión, letargia, aplasia, inapetencia y pérdida de peso; más antecedente de parasitismo por garrapatas. La mascota presentaba anemia leve $(4.730 .000 / \mathrm{mm} 3)$, enzimas hepáticas aumentadas (AST/ASA/GOT=72 $\mathrm{U} / \mathrm{I}$ ) y esplenomegalia. El test serológico in vitro y PCR para amplificar ADN E. canis fueron positivos, por lo que la mascota recibe tratamiento para EMC con doxiciclina $10 \mathrm{mg} / \mathrm{kg} /$ día durante 30 días, antiinflamatorios durante 5 días y protector hepático. A los 60 días de iniciado el tratamiento la mascota recuperó su peso normal y a los 120 días se realiza PCR como monitoreo de la eficacia del tratamiento dando resultado negativo.

SUMMARY. First report of canine monocytic ehrlichiosis in Santa Fe province, Argentina. Canine monocytic ehrlichiosis (CME) is a disease caused by Ehrlichia canis bacteria. It has a globally distributed and cause high mortality in domestic canines with nonspecific symptoms, which makes clinical diagnosis difficult. Eherlichia canis is transmitted to a host by the tick Rhipicephalus sanguineus sensu lato. In Argentina two lineages of this species are recognized (tropical and sensu stricto). The objective of this work is to report the first confirmed case of CME by E. canis in endemic area of $R$. sanguineus s.s from Rafaela, Santa Fe. On February 2, 2019 a canine arrived at the private clinic with non-specific symptoms such as late / night hyperthermia, depression, lethargy, aplasia, loss of appetite and weight loss, as well as, antecedent of tick parasitism. The pet had mild anemia $(4,730,000 / \mathrm{m} 3)$, increased liver enzymes (AST/ASA/GOT $=72 \mathrm{U} / \mathrm{I}$ ) and splenomegaly. The in vitro serological test and PCR to amplify $E$. canis DNA were positive, so the dog received treatment for CME with doxycycline $10 \mathrm{mg} / \mathrm{kg} /$ day for 30 days, anti-inflammatory for 5 days and liver protector. After 60 days of starting the treatment, the animal regained its normal weight and after 120 days the PCR have given negative result, checking the effectiveness of the treatment.

Palabras clave: ehrlichiosis monocitica canina, Ehrlichia canis, Rhipicephalus sanguineus, Santa Fe, Argentina

Keywords: Canine monocytic ehrlichiosis, Ehrlichia canis, Rhipicephalus sanguineus, Santa Fe, Argentina

La ehrlichiosis monocítica canina (EMC) es una enfermedad causada por la bacteria intracelular obligada Ehrlichia canis (Rickettsiales, Anaplasmataceae). La EMC es de importancia en salud animal por ser considerada de alta mortalidad en caninos domésticos, con distribución mundial (Harrus et al. 2012). Su periodo de incubación puede variar entre 8 y 20 días presen- tándose en tres fases: aguda, subclínica y crónica; con síntomas inespecíficos como fiebre alta, depresión, letargia, anorexia y signos clínicos como linfoadenomegalia, esplenomegalia y trombocitopenia con tendencia hemorrágica, por lo que la distinción de las tres fases es un desafío en el entorno clínico (Harrus y Waner, 2011). Ehrlichia canis es transmitida a un 
hospedador susceptible por ninfas y adultos de la garrapata Rhipicephalus sanguineus sensu lato (Neer et al. 2002). El taxón $R$. sanguineus s.l. representa un complejo de especies asociadas principalmente a caninos domésticos con distribución cosmopolita y particularmente en la región Neotropical se reconocen dos linajes: tropical y templado (Moraes-Filho et al. 2011; Nava et al. 2012, 2018). El linaje tropical ( $R$. sanguineus s.I.) se distribuye en áreas tropicales desde México al norte de Argentina y Chile, y el linaje templado, que representa a $R$. sanguineus sensu stricto, está asociado a localidades templadas y frías de Argentina, sur de Brasil, Chile y Uruguay, siendo el límite geográfico que separa ambos linajes una zona ecotonal situada entre $24^{\circ}$ y $25^{\circ}$ de latitud sur (Nava et al. 2012, 2018). En condiciones experimentales, $R$. sanguineus s.s. (poblaciones del centro de Argentina, Uruguay y sur de Brasil) demostró no tener competencia vectorial para transmitir E. canis (cepa Jaboticabal, Brasil) pero si $R$. sanguineus s.l. del linaje tropical ( $R$. sanguineus s.I. (LT)) (Moraes-Filho et al. 2015). Sin embargo, estudios recientes han determinado molecularmente la infección con $E$. canis en muestras de sangre de perros con diagnóstico presuntivo de EMC en la provincia de Buenos Aires (Eiras et al. 2013; Cicuttin et al. 2016), dónde prevalece $R$. sanguineus s.s. Además, poblaciones de $R$. sanguineus S.l. (LT) y $R$. sanguineus S.s. de las provincias de Formosa y Buenos Aires, respectivamente, se detectaron naturalmente infectadas con E. canis (Cicuttin et al. 2015, 2017). Cicuttin et al. (2017) plantean tres hipótesis para explicar cómo esta enfer-medad podría ser transmitida en áreas donde solo está presente $R$. sanguineus s.s. I) que los perros adquirieron la infección en áreas endémicas de $R$. sanguineus s.l. (LT) y la mantuvieron luego de su traslado de una ciudad a otra (la infección con Ehrlichia puede ser persistente en el hospedador vertebrado), y esto no implica que $R$. sanguineus s.s. sea el vector involucrado en los casos de Buenos Aires, II) que $R$. sanguineus s.s. posee competencia vectorial baja, pero no nula, o III) que $R$. sanguineus s.l (LT) ingresó a Buenos Aires con perros que migraron del norte Argentino y que se man-tuvieron durante la época climática favorable, no llegando a establecerse como poblaciones estables, pero suficiente para transmitir $E$. canis a hospedadores susceptibles locales.

El objetivo del presente trabajo es reportar el primer caso confirmado de EMC por E. canis en la provincia de Santa $\mathrm{Fe}$, Argentina, área endémica de $R$. sanguineus s.s.

El día 18/02/2019 llega a la consulta privada en la ciudad de Rafaela $\left(31^{\circ} 15^{\prime} \mathrm{S} 61^{\circ} 29^{\prime} \mathrm{O}\right.$ ), un canino de nombre Rocco, macho entero, de 9 años de edad y raza Braco, oriundo de dicha ciudad. El paciente padece hipotiroidismo bajo tratamiento con levotiroxina. Al momento de la consulta el animal presentaba signos de depresión, letargia, falta de apetito (selecciona alimen- to) e hipertermia tarde/noche. Al controlar el peso, el medico clínico corrobora que el animal había bajado 4 kilos. En la anamnesis el propietario indica que la mascota no tenía antecedentes de viajes fuera de la ciudad de origen y que en enero del mismo año se le había aplicado una pipeta para el control de pulgas, garrapatas y mosquitos (principio activo imidacloprid y permetrina) pero que a la semana de su aplicación se extrajo una garrapata viva prendida en piel. Se realiza hemograma completo, extendido sanguíneo de sangre periférica y control de funcionalidad hepática, arrojando como resultado leve anemia $\left(4.730 .000 / \mathrm{mm}^{3}\right)$, enzimas hepáticas aumentadas (AST/ASA/GOT=72 U/I) y a la observación directa del extendido sanguíneo, glóbulos rojos en pila de moneda (adhesión de los glóbulos rojos en cadena). Por este último método se descarta la presencia de Hepatozoon canis. No se realiza recuento plaquetario (ver Tabla 1).

El día 20/02/2019 el animal continúa con hipertermia tarde/noche por lo que el medico clínico decide internarlo. Se realiza ecografía abdominal la cual denota esplenomegalia.

El día 25/02/2019 el animal, continua con hipertermia tarde noche por lo que se realiza, hemograma, extendido sanguíneo, control de enzimas hepáticas y examen de orina. Arrojando valores normales en el hemograma y orina, enzimas hepáticas aumentadas (AST/ASA/GOT= $88 \mathrm{U} / \mathrm{l} ;$ ALT/ALAT/GPT= $131 \mathrm{U} / \mathrm{I})$, no se observa presencia de $H$. canis y no se realizó recuento plaquetario (tabla 1). Además, se realizó test comercial (Speed EhrliTM/ Virbac) para el diagnóstico serológico in vitro de E. canis, el cual arrojo resultado positivo. Dado los resultados obtenidos, comienza a administrarse al animal protector hepático. La mascota continúa con alimentación selectiva y decaimiento.

El día 28/02/2019 por la mañana la mascota comienza a convulsionar, siendo abolidas las contracciones por completo por la tarde. Se comienza a aplicar tratamiento para EMC con doxiciclina $10 \mathrm{mg} / \mathrm{kg} /$ día durante 30 días y antiinflamatorios durante 5 días para contrarrestar los síntomas (según Mylonakis et al. 2019). Ese mismo día se toma muestra de sangre con citrato para detección molecular directa de $E$. canis mediante la técnica de PCR (reacción en cadena de la polimerasa). La sangre fue sometida a extracción de ADN utilizando un kit comercial (Wizard genomic DNA/ Promega). A partir del ADN obtenido se amplificó, en primera instancia, mediante PCRrt (en tiempo real) un fragmento de $177 \mathrm{pb}$ del gen 16S ARNr de bacterias de la familia Anaplasmataceae (siguiendo a Monje et al. 2019), arrojando un resultado positivo. Para descartar infección con Anaplasma platys, el ADN fue sometido a PCR (convencional) amplificando un fragmento de $409 \mathrm{pb}$ del gen $d s b$ especifico de bacterias del género Ehrlichia (siguiendo a Doyle et al. 2005), dando resultado positivo. El fragmento $d s b$ amplificado fue secuenciado en la unidad de genómica del INTA Castelar. 
Tabla 1: Parámetros hematológicos y bioquímicos progresivos correspondientes al paciente canino (Rocco) con diagnóstico de Ehrlichiosis monocítica canina (EMC) en la ciudad de Rafaela, Santa Fe, Argentina. En negrita se resaltan valores anormales.

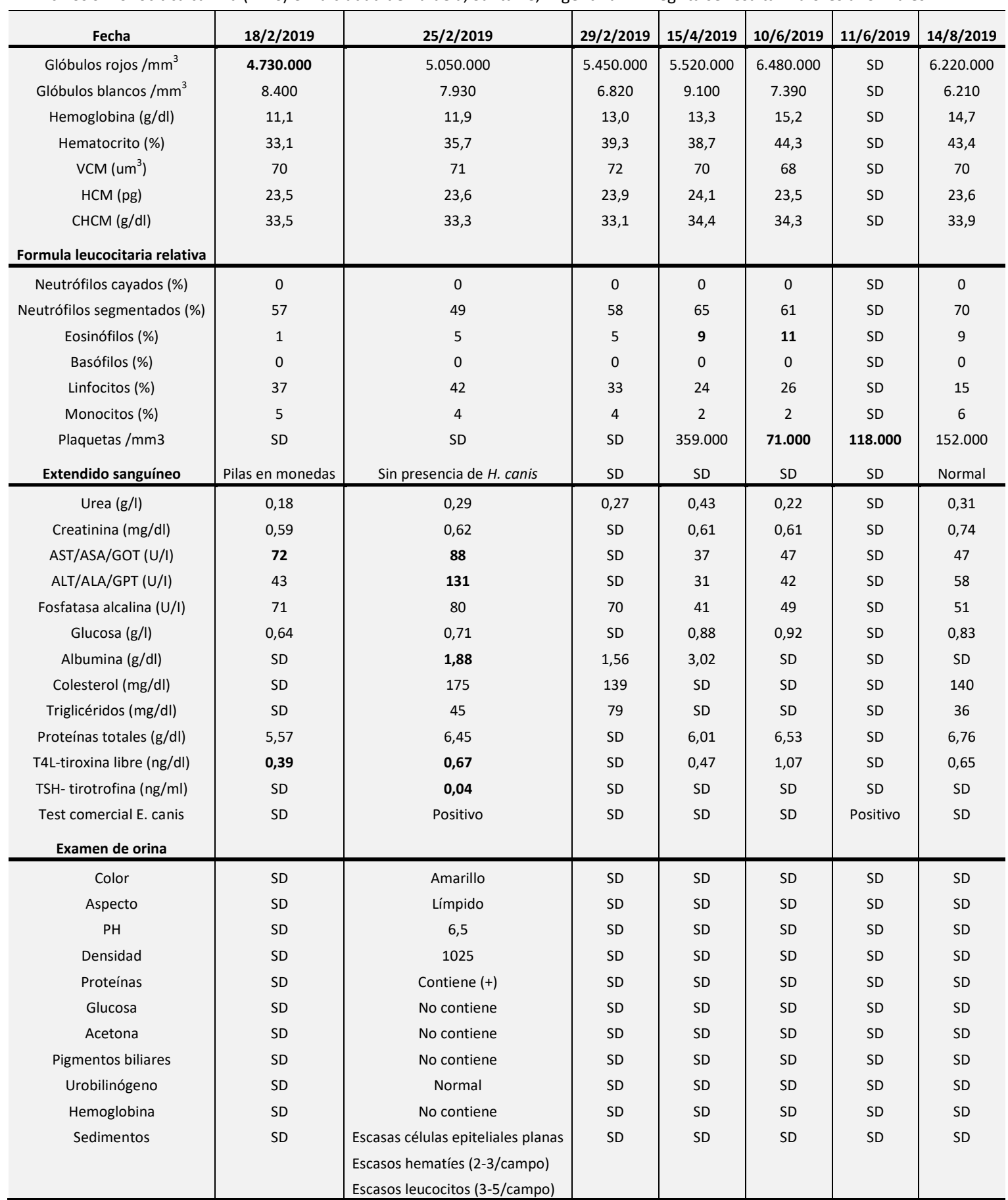

/mm3= milímetros cúbicos; $\mathrm{g} / \mathrm{dl}=$ gramos por decilitro; um3= micrómetro cubico; $\mathrm{pg}=$ picogramo; $\mathrm{g} / \mathrm{l}=$ gramos por litro; $\mathrm{mg} / \mathrm{dl}=\mathrm{miligramos}$ por decilitro; $\mathrm{U} / \mathrm{l}=$ unidades internacionales; $\mathrm{ng} / \mathrm{dl}=$ nanogramo por decilitro; $(\mathrm{ng} / \mathrm{dl})=$ nanogramo por mililitro. $\mathrm{SD}=\sin$ datos

La secuencia obtenida (Genbank MN617030) fue editada utilizando BioEdit Sequence Alignment Editor (Hall, 1999). Esta secuencia se comparó con secuencias depositadas en el GenBank, siendo $100 \%$ idéntica a las secuencias de $d s b$ de $E$. canis aisladas de perros en Argentina, Brasil, Estados Unidos, Cameron, Colombia y Tailandia (№ Genbank: KU253450, GU586135, CP000107, DQ124254, MK783026, KY576856), así como también $100 \%$ idéntica a las secuencias de E. canis detectadas en $R$. sanguineus s.I. (LT) y $R$. sanguineus s.s. de Argentina (№ Genbank: KR909452, MF805005). Del análisis filogenético se obtuvo un árbol de máxima verosimilitud (ML) construido a partir de secuencias de gen $d s b$ de distintas especies de Ehrlichia (modelo de sustitución: Tamura 3 parámetros $+\mathrm{G}$ ). Denotando una inexistencia de polimorfismo genético entre las distintas cepas de $E$. canis de distribución mundial para el gen $d s b$ de tipo conservado (Figura 1). Adicionalmente, se 
amplificó un fragmento de $935 \mathrm{pb}$ del gen 18S ARNr (siguiendo a Thompson et al. 2018) para descartar coinfección con Babesia vogeli arrojando resultado negativo.

El día 15/04/2019 se realiza control clínico del paciente, corroborándose que la mascota recupero su peso normal en 60 días. Se realiza hemograma completo de control, arrojando valores normales con leve aumento de eosinófilos (9\%). Recuento plaquetario y enzimas hepáticas normales (Tabla 1).

El día 10/06/2019 se realiza hemograma completo de control, con valores normales con leve aumento de eosinófilos (11\%). Recuento plaquetario marcadamente por debajo de los valores normales $\left(71.000 / \mathrm{mm}^{3}\right)$.

El día 11/06/2019 se repite recuento plaquetario arrojando un leve aumento de plaquetas circulantes, pero por debajo de lo normal $\left(188.000 / \mathrm{mm}^{3}\right)$. Además, se realiza test serológico comercial para diagnóstico de $E$. canis, el cual arrojó resultado positivo. El día 14/08/ 2019 se realiza control del paciente y análisis de laboratorio, los que arrojan resultado dentro de los parámetros normales (ver tabla 1). Se realiza PCR amplificando el gen $d s b$, como monitoreo de la eficacia del tratamiento, el cual arrojó resultado negativo.

El presente informe de caso corresponde al primer diagnóstico confirmado de EMC por E. canis en la provincia de Santa $\mathrm{Fe}$, en un área de distribución de $R$. sanguineus s.s. Dada la inespecificidad de síntomas y signos con los que se desarrolla esta enfermedad, es difícil alcanzar un diagnóstico clínico preciso, teniendo en cuenta sus similitudes con otras enfermedades transmitidas por garrapatas como ser babesiosis o hepatozoonosis canina. Esto hace necesario que la EMC sea considerado como diagnostico presuntivo hasta tanto no sea confirmada mediante métodos directos, como la observación en extendido sanguíneo de la forma visible denominada mórula (estadio morfológico de E. canis dentro del fagosoma de la célula diana), la detección molecular de ADN bacteriano mediante la técnica de PCR, o por métodos indirectos, mediante detección de anticuerpos contra E. canis por serología (Neer et al. 2002; Nosach et al. 2018). Si bien en el caso aquí reportado al comienzo de la enfermedad se presenta una leve anemia, es prudente mencionar que algunos autores sugieren que pacientes infectados pueden presentar recuentos de eritrocitos normales (Neer et al. 2002). Así también, la trombocitopenia característica de esta enfermedad (ocurre en el $82 \%$ de los casos) puede no desarrollarse al comienzo de la misma (Nosach et al. 2018), en el caso clínico aquí reportado, no se realizaron recuentos plaquetarios al comienzo, y aunque, pudo observarse una trombocitopenia marcada incluso a meses de finalizado el tratamiento, esto último podría atribuirse a otras causas y no a EMC por E. canis. Algunos autores, para casos de EMC en Estados Unidos aseguran que usualmente la trombocitopenia debería resolverse a los 14 días de iniciado el tratamiento e incluso es un indicativo de una correcta respuesta del paciente al tratamiento (Neer et al. 2002). Nuestro paciente, además, mostro hipoalbuminemia, lo cual se asocia a la perdida por vía renal, intestinal, hepática y a la mala nutrición, así como la elevación de las enzimas hepáticas, dependiendo de la afectación orgánica.

Es cauto destacar que en la mayoría de los casos de EMC no es posible la observación de mórulas en extendidos sanguíneos. Algunos autores estiman que solo en el $4 \%$ de las formas agudas de los casos es factible su hallazgo (Harrus y Waner, 2011). Es en este punto donde se hace necesario poder confirmar el diagnóstico mediante amplificación de ADN bacteriano por técnica de PCR, lo que además de caracterizar el agente etiológico, permite descartar coinfecciones con otros microorganismos de la familia Anaplasmataceae, como es el caso de $A$. platys o protozoarios como $B$. vogeli o $H$. canis. Algunos autores sugieren que las manifestaciones clínicas de EMC pueden exacerbarse cuando otros patógenos transmitidos por vectores están presentes (Little, 2010). Eiras et al. (2013) reportan en la provincia de Buenos Aires, una alta prevalencia de coinfección en casos clínicos de EMC por E. canis con $\mathrm{H}$. canis. Asimismo, pueden darse resultados falsos negativos por PCR cuando $E$. canis se acantona en órganos como bazo, hígado y medula ósea (Harrus y Waner, 2011). Es importante remarcar que el test diagnostico serológico comercial, es una herramienta rápida en la clínica cotidiana, pero utilizar solo este método para confirmar casos sospechosos puede ocasionar una sobrestimación de EMC en la región (Cicuttin et al. 2017), pudiendo arrojar falsos positivos al producirse reacción cruzada con Ehrlichia ewingii, Ehrlichia chaffeensis o A. platys (Neer et al. 2002) o falsos negativos al no detectarse anticuerpos en sangre en fase aguda. Se ha reportado que, en algunos casos, habiéndose resuelto el cuadro clínico, la persistencia de elevados títulos de anticuerpos (por inmunofluorescencia indirecta) se observa por años (Neer et al. 2002).

No se pudo asociar el desarrollo de este caso de EMC con una especie de garrapata vector, dado que, si bien el dueño informó al clínico la presencia de una garrapata no se tuvo acceso a la misma.

Si bien en este estudio no se observaron diferencias entre las secuencias del gen $d s b$ obtenidas de diferentes cepas de $E$. canis originarias de distintas áreas geográficas, dado que, el gen $d s b$ es apropiado para el diagnóstico, pero su carácter conservado no permite la diferenciación genética de las diferentes cepas dentro de la especie (figura 1). Es así que, se resalta la necesidad de realizar futuros estudios comparando genes con un mayor nivel de polimorfismo, a fin de evaluar variaciones genéticas intraespecíficas y determinar si la gravedad de los casos clínicos de EMC podría estar vinculada con la virulencia de la cepa infectante de $E$. canis. 
Para comprender la epidemiología, evaluar potenciales riesgos y diseñar estrategias de control de EMC por $E$. canis en la región centro de Argentina, se considera necesario realizar futuros esfuerzos a fin de lograr el aislamiento, caracterización y cultivo de esta cepa y posterior evaluación de la competencia vectorial de $R$. sanguineus s.s. para transmitirla.

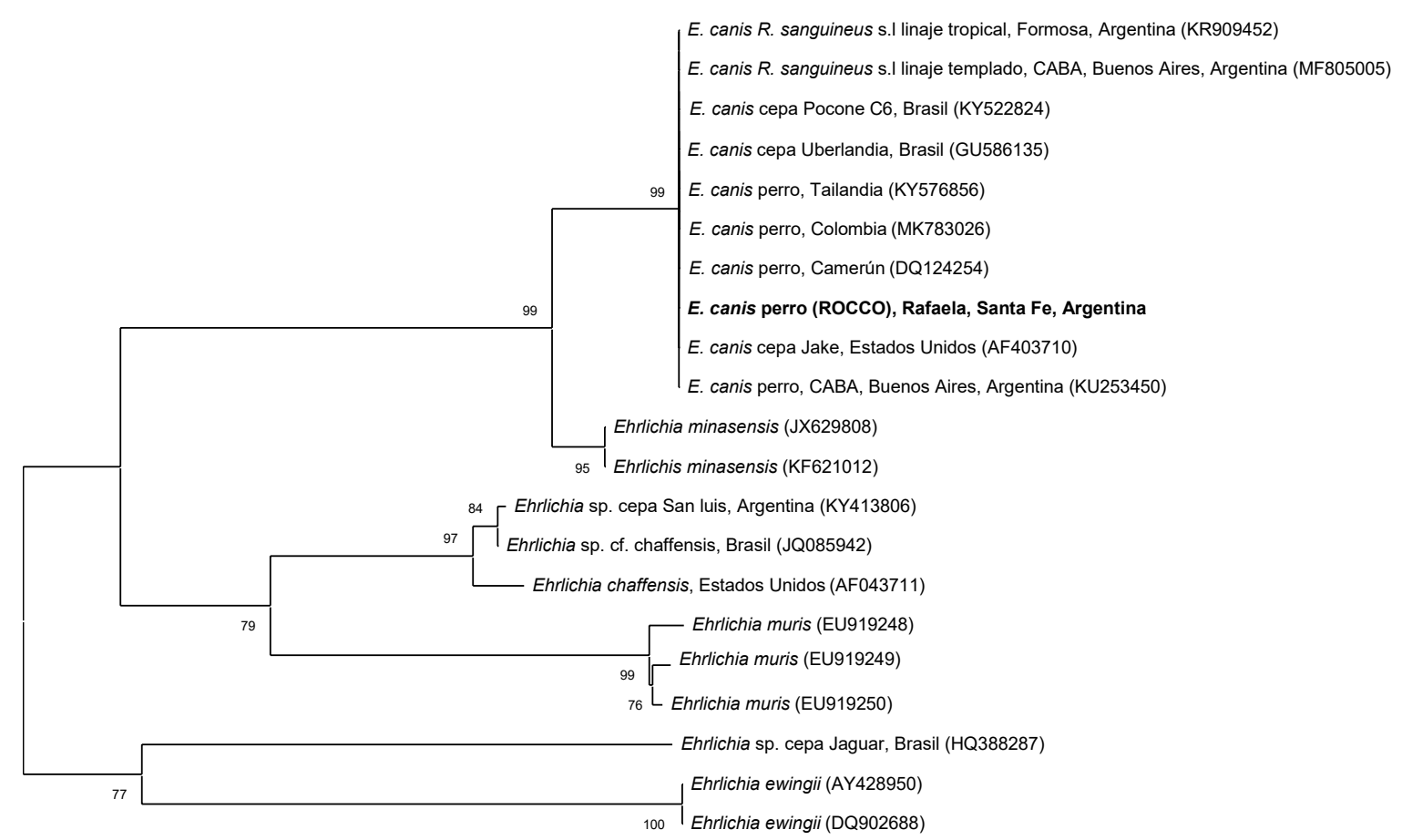

0.050

Figura 1: Árbol de máxima verosimilitud (ML) construido a partir de secuencias de gen $d s b$ de distintas especies de Ehrlichia (modelo de sustitución: Tamura 3 parámetros $+\mathrm{G}$ ). Los números representan el soporte de arranque generado a partir de 1000 repeticiones. Entre paréntesis, los números de acceso de GenBank. En negrita, se resalta Ehrlichia canis detectada en sangre del paciente con diagnóstico de Ehrlichiosis monocítica canina de la ciudad de Rafaela, Santa Fe, Argentina.

\section{Agradecimientos}

Los autores agradecen el apoyo financiero de INTA-EEA Rafaela, Asociación Cooperadora INTA Rafaela (ELT, NA, SN) y Fundación Alberto J. Roemmers Subsidio 2018 "Epidemiología de microorganismos Rickettsiales (Rickettsia, Ehrlichia y Anaplasma), asociados a garrapatas (Acari: ixodidae), de importancia sanitaria en Argentina" (ELT, FSF, LM).

\section{Bibliografía}

Cicuttin GL, De Salvo MN, Gury Dohmen FE. 2016. Molecular characterization of Ehrlichia canis infecting dogs, Buenos Aires. Ticks Tick-Borne Dis. 7: 954-957.

Cicuttin GL, Tarragona EL, De Salvo MN, Mangold AJ, Nava S. 2015. Infection with Ehrlichia canis and Anaplasma platys (Rickettsiales: Anaplasmataceae) in two lineages of
Rhipicephalus sanguineus sensu lato (Acari: Ixodidae) from Argentina. Ticks Tick-Borne Dis. 6: 724-729.

Cicuttin G, De Salvo MN, Silva DA, Brito M, Nava S. 2017. Ehrlichia canis (Rickettsiales: Anaplasmataceae) en garrapatas Rhipicephalus sanguineus sensu lato del linaje templado (Acari. Ixodidae), provincia de Buenos Aires, Argentina. FAVE Cs. Vet. 16: 93-96.

Doyle CK, Labruna MB, Breitschwerdt EB, Tang $\mathrm{Y}$, Corstvet RE, Hegarty BC, Bloch KC, Li P, Walker DH, McBride JW. 2005. Detection of medically important Ehrlichia by quantitative multicolor TaqMan real-time PCR of the dsb Gene. J. Mol. Diagn. 7: 504-510.

Eiras DF, Craviotto MB, Vezzani D, Eyal O, Baneth G. 2013. First description of natural Ehrlichia canis and Anaplasma platys infections in dogs from Argentina. Comp. Immunol. Microbiol. Inf. Dis. 36: 169-173.

Hall TA. 1999. BioEdit: a user friendly biological sequence alignment editor and analysis program for Windows 95/98/NT. Nucl. Acids Symp. 41: 95-98. 
Harrus S, Waner T. 2011. Diagnosis of canine monocytotropic ehrlichiosis (Ehrlichia canis): an overview. Vet. J. 187: 292-296.

Harrus S \& Waner T, Neer TM. 2012. Ehrlichia canis infection. En: Greene CE (eds.). Infectious Diseases of the Dog and Cat, Elsevier, Missouri. Pp. 227-238.

Little SE. 2010. Ehrlichiosis and anaplasmosis in dogs and cats. Vet. Clin. North Am. Small Anim. Pract. 40: 1121-1140.

Moraes-Filho J, Marcili A, Nieri-Bastos F, Richtzenhain LJ, Labruna MB. 2011. Genetic analysis of ticks belonging to the Rhipicephalus sanguineus group in Latin America. Acta Trop. 117: 51-55.

Moraes-Filho J, Krawczak FS, Costa FB, Soares JF, Labruna MB. 2015. Comparative evaluation of the vector competence of four South American populations of the Rhipicephalus sanguineus Group for the bacterium Ehrlichia canis, the agent of Canine Monocytic Ehrlichiosis. Plos One 10: e0139386.

Monje LD, Fernandez C, Percara A. 2019. Detection of Ehrlichia sp. strain San Luis and Candidatus Rickettsia andeanae in Amblyomma parvum ticks. Ticks Tick-Borne Dis. 10: 111-114.

Mylonakis ME, Harrus S, Breitschwerdt EB. 2019. An update on the treatment of canine monocytic ehrlichiosis (Ehrlichia canis). Vet. J. 246: 45-53.

Nava S, Mastropaolo M, Venzal JM, Mangold AJ, Guglielmone AA. 2012. Mitochondrial DNA analysis of Rhipicephalus sanguineus sensu lato (Acari:Ixodidae) in the Southern Cone of South America. Vet. Parasitol. 190: 547-555.

Nava S, Beati L, Venzal JM, Labruna MB, Szabó MP, Petney T, Saracho-Botterop MN, Tarragona EL, Dantas-Torres F, Santos Silva MM, Mangol AJ, Guglielmone AA, Estrada-Peña A. 2018. Rhipicephalus sanguineus (Latreille, 1806): neotype designation, morphological re-description of all parasitic stages and molecular characterization. Ticks Tick-Borne Dis. 9: 15731585.

Neer TM, Breitschwerdt EB, Greene RT, Lappin MR. 2002. Consensus statement on ehrlichial disease of small animals from the infectious disease study group of the ACVIM. J. Vet. Intern. Med. 16: 309-315.

Nosach N, Vesco C, Regonat M, Vartabedian A. 2018. Ehrlichia canis: revisión bibliográfica. Rev. Vet. 35 (368): 1-13.

Thompson CS, Mangold AJ, Félix ML, Carvalho L, ArmúaFernández MT, Venzal J.M. 2018. Molecular evidence of Babesia species in Procyon cancrivorus (Carnivora, Procyonidae) in Uruguay. Vet. Parasitol. Reg. Stud. Reports 13: 230-233. 